\title{
Matraca: Ferramenta Computacional para Auxílio à Inclusão Digital de Deficientes Visuais
}

\section{Matraca: Computational Tool for digital accessibility of visually impaired}

\author{
Delano Hélio Oliveira ${ }^{1}$ \\ delanohelio@gmail.com \\ Izabela Vanessa de Almeida Melo ${ }^{1}$ \\ izabela.vmelo@gmail.com \\ Natã Venâncio de Melo ${ }^{1}$ \\ nata.venancio.melo@gmail.com \\ Joseana Macêdo Fechine ${ }^{1}$ \\ joseana@dsc.ufcg.edu.br \\ ${ }^{1}$ Universidade Federal de Campina Grande
}

Resumo. No mundo moderno, observa-se cada vez mais a presença da informática na vida cotidiana. Dentro desse contexto, insere-se o Matraca, uma ferramenta open source, de fácil uso, com exigência de poucos recursos computacionais, cujo principal objetivo é promover a inclusão digital de pessoas com deficiência visual. A ferramenta é composta por três aplicativos úteis em tarefas básicas relacionadas ao uso de um computador: um editor de texto, um sistema para envio de mensagem eletrônica e uma calculadora. A interface possui elementos gráficos especiais e elementos sonoros (de entrada e saída) que visam facilitar ainda mais a interação do usuário com a máquina. Atualmente, a ferramenta se encontra em fase de testes para posterior avaliação formal da usabilidade, a qual será conduzida pelos usuários do Instituto dos Cegos de Campina Grande, instituição parceira do projeto. 
Palavras-chave: inclusão digital, deficientes visuais, Matraca, ferramenta open source.

\begin{abstract}
In the current world we have seen a massive presence of the technology in the people's life. In this context the Matraca is inserted. This is an open source tool have how main goal provide digital inclusion for people with visual disability. The tool is composite for three applications: a text editor, an email system and a calculator. The interface has specials graphics and sounds effects (to in and out). Stands yet that tool should provide a mechanism to turn possible its use in user mode (reality to use in lan-house). In the work actual stage are being realized adjustments in the tool for turn better its performance. In the next stage, the people will test the Matraca for help detect fails and finally can offer this tool for the public.
\end{abstract}

Keywords: digital inclusion, visually impaired, Matraca, open source tool

\title{
1. Introdução e conteúdos
}

Uma estimativa, aceita pelos institutos de pesquisa e pelos principais órgãos de referência em saúde, revela que existem 6,5 bilhões de seres humanos e que 10 a $12 \%$ da população possuem algum tipo de deficiência física ou intelectual. No Brasil, 14,5\% da população são pessoas portadoras de deficiência e, aproximadamente, 6,5 milhões possuem algum grau de deficiência visual (IBGE, 2005). Nesse contexto, insere-se a Tecnologia Assistiva que visa promover a inclusão.

Tecnologia Assistiva é um termo utilizado para referenciar o conjunto de recursos e serviços que visam melhorar as habilidades de pessoas deficientes (ADA, 1990; LAUAND, 2005). Os recursos têm como finalidade aumentar, melhorar ou manter as capacidades funcionais dessas pessoas. Os recursos podem ser qualquer item, equipamento ou produto, desde uma simples bengala a um hardware ou software sofisticado, equipamentos que contemplam questões de acessibilidade. Os serviços são aqueles prestados profissionalmente à pessoa com deficiência, buscando selecionar, comprar ou usar instrumentos de Tecnologia Assistiva (os recursos). Esses serviços 
envolvem profissionais de diversas áreas, como: Fonoaudiologia, Medicina, Engenharia, Arquitetura, dentre outras.

O objetivo da Tecnologia Assistiva é, portanto, proporcionar à pessoa portadora de deficiência maior independência, qualidade de vida e inclusão social, a partir da ampliação da comunicação, mobilidade, controle do seu ambiente, habilidades de seu aprendizado, competição, trabalho e integração com a família, amigos e sociedade (ADA, 1990; LAUAND, 2005).

No mundo atual, é extremamente necessário que haja inclusão digital de todas as pessoas, mais especificamente no uso do computador como ferramenta para o lazer, no auxílio à educação, na aquisição de informações e em diversas outras atividades. Contudo, isso se torna mais difícil quando se trata de pessoas portadoras de deficiência visual, que necessitam de ferramentas especiais para utilização do computador.

Atualmente, mesmo com os leitores de tela, a acessibilidade dos deficientes visuais (DV) a um computador não é plena (ABEDEV, 2002; SONZA, 2004). A maioria dos leitores disponíveis necessita ser instalado, o que seria análogo a instalar e configurar um sistema operacional toda vez que o DV fosse utilizar um computador diferente. Essa instalação nem sempre é permitida a um usuário (a exemplo de um DV) em uma lan house. Dessa forma, o acesso de um DV a um computador pode se tornar limitado. Destaca-se, também, que alguns leitores de tela de qualidade são pagos, com preço elevado e necessitam de recursos consideráveis de hardware e de software.

Para tornar a inclusão digital mais efetiva, permitindo a execução de algumas tarefas no computador, por deficientes visuais, torna-se imperativo o uso de uma ferramenta com componentes especiais que torne mais simples e acessível o uso da máquina por estas pessoas. O software Matraca se insere nesse contexto, um recurso de acessibilidade ao computador, de fácil uso e com poucas exigências de recursos de hardware e de software, útil para cegos ou pessoas de baixa visão.

Visando atender as necessidades supracitadas, o software Matraca é Open Source, de baixo custo computacional e composto por três aplicativos: um editor de texto, um sistema para envio de mensagem eletrônica e uma calculadora. Esta ferramenta possibilita, portanto, ao deficiente visual utilizar aplicativos simples, que são bastante úteis para o seu dia-a-dia. 


\section{Metodologia}

A metodologia adotada para o desenvolvimento da ferramenta Matraca consistiu em uma subdivisão em etapas, descrita a seguir:

1. Observação e identificação de limitações de softwares utilizados atualmente por deficientes visuais;

2. Estudo das dificuldades encontradas pelos deficientes visuais quando da realização de atividades a partir do uso de computadores;

3. Estudo do estado da arte sobre ferramentas computacionais de auxílio a deficientes visuais no uso do computador e de conceitos que possam ser incluídos no Projeto Matraca;

4. Implementação, testes e Implantação da ferramenta.

O processo de desenvolvimento de software utilizado foi o XP1 (SAUVÉ, 2007), com definição de requisitos funcionais e não funcionais baseada nas necessidades obtidas em reuniões com usuários do Instituto dos Cegos de Campina Grande, instituição parceira do projeto (ICEGOS, 1952).

\section{Resultados e discussões}

O Matraca é um software Open Source, implementado na linguagem de programação Java, incluindo um editor de texto, uma calculadora e um sistema para envio de mensagem eletrônica, desenvolvido especialmente para deficientes visuais.

Além de Open Source, o Matraca é também gratuito e seus direitos legais estão de acordo com a GPL (GNU General Public License) (GNU, 2007). Assim, os usuários não têm custos para a utilização do software, tornando-o acessível a todos. Além disso, como o código é aberto, qualquer membro de comunidades de apoio ao software livre pode modificá-lo, agregando novos conceitos e funcionalidades, com o objetivo de melhorá-lo. 


\subsection{Visão Geral do Matraca}

Na Figura 1, é apresentada uma visão geral da arquitetura do Matraca.

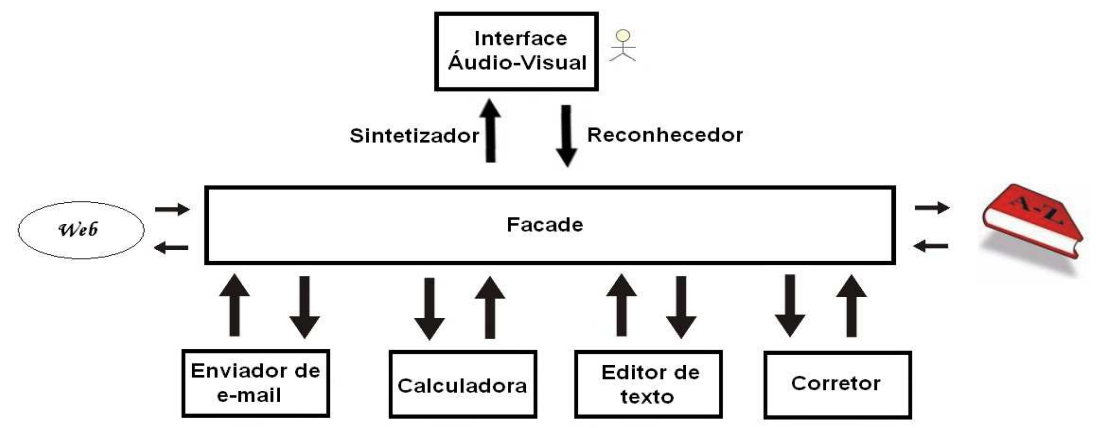

Figura 1 - Visão geral da arquitetura do Matraca.

Observa-se, a partir da Figura 1, que existe uma camada denominada Facade que possibilita a comunicação entre os diversos componentes do Matraca. O Enviador de e-mail, a Calculadora, o Editor de texto e o Corretor ortográfico trocam informações com o Facade e este, por sua vez, efetua a troca de informações com a Web, Banco de Dados e a Interface Áudio-Visual e vice-versa. Desta forma, o Matraca executa suas funcionalidades e realiza a comunicação com o usuário.

Considerando que o Matraca será utilizado, preferencialmente, por deficientes visuais (cegos), o principal meio de comunicação, entre o software e o usuário, é a fala, a partir da síntese de voz (comunicação software - usuário) e do reconhecimento automático de fala (comunicação usuário - software) (LEMMETTY, 2000, KLEIJN, 1995, RABINER, 1978, O'SHAUGHNESSY, 2003). Para a síntese de voz, foi utilizado o FreeTTS, sintetizador gratuito para Java, implementado a partir do framework JSAPI (Java Speech API) (FreeTTS, 2005). Todas as informações disponíveis ao usuário, por meio do menu do software, também podem ser acessíveis por meio do sintetizador de voz. Para o reconhecimento automático de fala, foi utilizado o Sphinx4, reconhecedor automático de fala gratuito para Java (SPHINX4, 2010). O reconhecedor é utilizado em conjunto com um corretor ortográfico automático e permite ao usuário escolher uma dentre cinco opções de palavras disponíveis, 
sendo suficiente, para tanto, o uso de um reconhecedor automático de dígitos isolados (dígitos de zero a cinco).

Considerando a exigência do uso de software gratuito, estão sendo utilizados atualmente sintetizador e reconhecedor apenas para a língua inglesa, dado que os disponíveis para a língua portuguesa de boa qualidade não são gratuitos. Porém, este fato não minimiza o uso da interação de voz disponibilizada pela ferramenta, visto que uma parcela considerável dos usuários já utiliza outras ferramentas que interagem a partir da língua inglesa, a exemplo do JAWS (2010). Mesmo assim, estudos estão sendo realizados visando ampliar o uso também para a língua portuguesa.

\subsection{Descrição e Apresentação do Matraca}

O Matraca possibilita a execução de três ações: edição de texto, realização de cálculos e envio de mensagens eletrônicas. Destaca-se, também, que a ferramenta disponibiliza imagens intuitivas, com tamanho destacado e mensagens de áudio (utilizando sintetizador de voz e reconhecedor de dígitos isolados), visando facilitar a sua utilização por deficientes visuais.

A tela inicial do Matraca (Figura 2) possui três botões com tamanho destacado e com imagens intuitivas para facilitar o uso por deficientes visuais e um botão para finalizar o sistema. Ao passar o mouse sobre os botões, é pronunciada a função correspondente. Por exemplo, são pronunciados os textos "Calculator", "Email system", "Text editor" e "Close the system" ao passar o mouse sobre os botões da Calculadora, do Email, do Editor de texto e do botão de fechar o sistema, respectivamente.

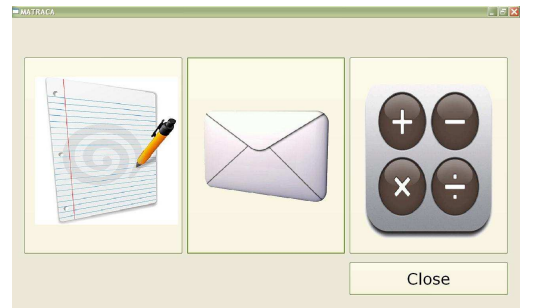

Figura 2 - Tela inicial do Matraca (Editor de Texto, Envio de e-mail e calculadora, respectivamente). 
$\mathrm{O}$ editor de textos do Matraca permite que o usuário digite, escute, corrija e salve um texto, abra um arquivo existente em disco e envie por $e$-mail o texto presente no editor. Além disso, o ambiente disponibiliza dois botões de ajuda ao usuário, sendo um deles para a elocução de todos os atalhos existentes no editor. É possível observar, a partir da Figura 3, que o editor de texto possui áreas reservadas para: os botões indicativos das funcionalidades, para a digitação do texto e para a correção das palavras digitadas.

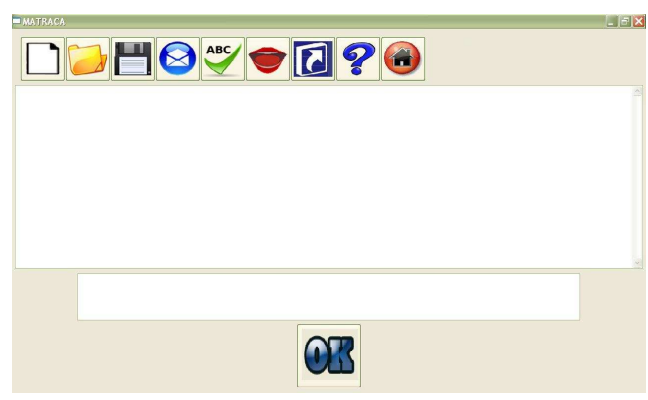

Figura 3 - Editor de texto do Matraca.

O corretor de texto é executado em paralelo à digitação do usuário. A cada espaço dado no editor, uma palavra digitada é analisada. Se a palavra estiver com a grafia errada, um som é emitido, e opções para correção aparecem na tela abaixo do local onde o usuário digita. Para corrigir, o usuário pode utilizar as setas para baixo e para cima para selecionar uma palavra - cada opção selecionada é pronunciada pelo sintetizador de voz do Matraca - e depois dá-se um ESPAÇO para efetuar a correção. Pode-se também fazer a escolha utilizando o mouse ou a partir da elocução de dígitos, de forma isolada, em que cada dígito indica qual a opção desejada.

Os passos para a correção de uma palavra digitada de forma incorreta são apresentados na Figura 4.
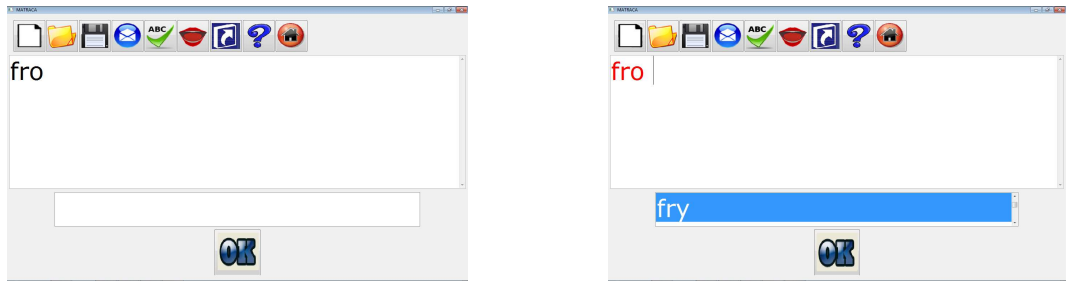

V. $9 \mathrm{~N}^{\mathrm{o}}$ 1, julho, 2011 
Figura 4.a - Digitação incorreta de uma palavra.
Figura 4.b - Indicação de sugestões para correção.

No exemplo, tenta-se digitar a palavra "for", mas, por engano, digitou-se "fro" (Figura 4.a). O Matraca detecta o possível erro, um alerta sonoro é emitido e são apresentadas, no campo de correção, as sugestões de opções de palavras semelhantes, recuperadas do dicionário (Figura 4.b). Essas opções são pronunciadas uma a uma de acordo com a passagem de uma tecla (up ou down) ou do mouse sobre as palavras. Ao escolher uma das opções, pressiona-se o botão OK ou um ESPAÇO e a palavra selecionada substituirá a palavra marcada no editor. Se o usuário desejar que a palavra marcada tenha a correção ignorada, utiliza-se a opção "Ignore Word”. A palavra também poderá ser adicionada ao dicionário escolhendo-se a penúltima opção pronunciada, que corresponderá à referida palavra.

Além do editor de textos, o Matraca também disponibiliza uma calculadora que realiza operações de soma, subtração, multiplicação e divisão. Seus botões apresentam tamanho destacado e ao passar do mouse sob o botão, o sintetizador de voz pronuncia o número, operação ou sua função, facilitando, assim, o uso da ferramenta por um DV. O sintetizador também pronuncia o resultado da operação. A calculadora é de fácil uso e possui botões com tamanho destacado, conforme ilustrado na Figura 5, a qual exemplifica o cálculo da operação 39 x 2, cujo resultado é 78.0.

\begin{tabular}{|c||c|c|c|}
\hline 78.0 \\
\hline 7 & 8 & 9 & + \\
\hline 4 & 5 & 6 & - \\
\hline 1 & 2 & 3 & $*$ \\
\hline 0 & $\cdot$ & $=$ & $/$ \\
\hline
\end{tabular}

Figura 5 - Resultado da operação 39 x 2 na calculadora do Matraca.

O ambiente para envio de mensagens eletrônicas (email) também é bastante intuitivo. Conforme Figura 6, há espaços para digitação do email do remetente e do destinatário, assunto, senha do 
remetente (para permitir acesso à conta de envio) e a mensagem. Estão disponíveis botões para enviar (Send), apagar tudo (Clean) e falar (Speak) as informações digitadas na tela (exceto a senha). A ferramenta permite o uso dos servidores de email do Gmail, Hotmail e Yahoo!Mail, podendo ser expandida para outros servidores. A exemplo das demais funcionalidades, botões e espaços para digitação são pronunciados pelo sintetizador de voz.

Pode-se observar, portanto, que o Matraca possibilita, ao deficiente visual, utilizar aplicativos de forma rápida e intuitiva.

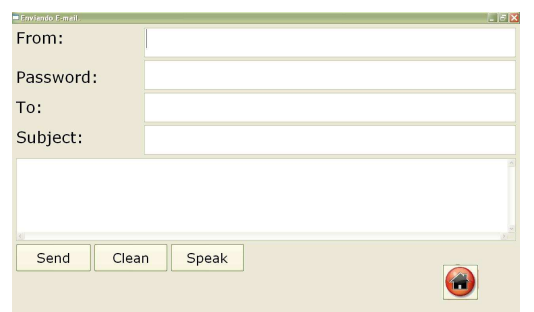

Figura 6 - Ambiente para envio de email.

\section{Conclusões e trabalhos futuros}

O Matraca é uma ferramenta que auxilia deficientes visuais em atividades comuns relacionadas ao uso do computador, além de ser uma ferramenta gratuita e de fácil uso. Visando tornar a ferramenta ainda mais eficiente, novas funcionalidades serão implementadas, para que o Matraca se torne um software portátil e que possibilite ao usuário a escolha do idioma a ser usado (português ou inglês). O passo seguinte, e de suma importância para o uso do Matraca, será a sua instalação e testes no Instituto dos Cegos de Campina Grande.

O Matraca se constitui, portanto, uma ferramenta computacional que auxilia o deficiente visual em atividades do cotidiano de maneira prática, melhorando o seu dia-a-dia, seja por intermédio do acesso à educação ou até na conquista de oportunidades no mercado de trabalho, auxiliando, assim, na promoção da inclusão digital. 


\section{Referências}

ABEDEV - Associação Brasileira de Educadores de deficientes visuais. Disponível em http://intervox.nce.ufrj.br/ abedev/ ApostilaDV.doc. Acesso em: 04 de julho de 2011.

ADA. AMERICANS WITH DISABILITIES ACT OF 1990, AS AMENDED. Disponível em: http://www.ada.gov/pubs/ada.htm. Acesso em: 04 julho 2011.

FreeTTS. FreeTTS 1.2 - A speech synthesizer written entirely in the $\mathrm{Java}^{\mathrm{TM}}$ programming language. Disponível em: http://freetts.sourceforge.net. Acesso em: 04 julho 2011.

GNU. GNU General Public License. Disponível em: http://www.gnu.org/licenses/gpl.html.Acesso em: 04 julho 2011.

IBGE. IBGE e CORDE: Encontro internacional de estatísticas sobre pessoas com deficiência. Disponível em: http://www.ibge.gov.br/home/presidencia/noticias/noticia_visualiza.php? id_noticia=438\&id_pagina=1. Acesso em: 04 julho 2011.

ICEGOS. Instituto dos Cegos de Campina Grande - PB. Disponível em: http://www.institutodoscegos.com.br/. Acesso em: 04 julho 2011.

JAWS. JAWS for Windows Screen Reading Software Disponível em: http://www.freedomscientific.com/product-portal.asp. Acesso em: 04 julho 2011.

KLEIJN, W.B; PALIWAL, K. K. Speech Coding and Synthesis. New York: Elsevier Science Inc, 1995.

LAUAND, G. B. A. Fontes de informação sobre tecnologia assistiva para favorecer a inclusão escolar de alunos com deficiências físicas e múltiplas. Tese de Doutorado em Educação Especial, Programa de Pós-Graduação em Educação Especial, Universidade Federal de São Carlos. 2005.

LEMMETTY, S. Review of Speech Synthesis Technology. Disponível em: http://www.acoustics.hut.fi/publications/ files/theses/lemmetty_mst/chap1.html.Acesso em: 04 julho 2011. 
RABINER, L. R. and SCHAFER W. R. (1978). Digital Processing of Speech Signals, Prentice Hall.

SONZA, A. P. Acessibilidade de deficientes visuais aos ambientes digitais/virtuais. Dissertação de Mestrado, Programa de PósGraduação em Educação. Faculdade de Educação. Universidade Federal do Rio Grande do Sul. 2004.

SPHINX4. Sphinx-4 - A speech recognizer written entirely in the $\mathrm{Java}^{\mathrm{TM}}$ programming language. Disponível em: http://cmusphinx.sourceforge.net. Acesso em: 04 julho 2011.

O'SHAUGHNESSY, D., Interacting with Computers by Voice: Automatic Speech Recognition and Synthesis, Proceedings of the IEEE, 91 (9), Setembro. 2003.

SAUVÉ, J. P.; NETO, E. R; FEITOSA, A. L.; DA SILVA, A. F.; NETO, F. R. de A. e CABRAL, F. W. L. XP1: Um Processo de Desenvolvimento. Disponível em: http://www.dsc.ufcg.edu.br/ jacques/projetos/common/xp1/xp1.html. Acesso em: 04 julho 2011. 